\title{
Near-infrared observations of galaxies in Pisces-Perseus
}

\section{I. $H$-band surface photometry of 174 spirals $^{\star}$}

\author{
G. Moriondo ${ }^{1}$, C. Baffa ${ }^{2}$, S. Casertano ${ }^{3}$, G. Chincarini ${ }^{4}$, G. Gavazzi ${ }^{5}$, C. Giovanardi ${ }^{2}$, L.K. Hunt $^{6}$, \\ D. Pierini ${ }^{7}$, M. Sperandio ${ }^{4}$, and G. Trinchieri ${ }^{8}$
}

1 Dipartimento di Astronomia e Scienza dello Spazio, Università di Firenze, Largo E. Fermi 5, I-50125 Firenze, Italy

2 Osservatorio Astrofisico di Arcetri, Largo E. Fermi 5, I-50125 Firenze, Italy

3 STECF Space Telescope Science Inst., 3700 San Martin Drive, Baltimore, MD 21218, U.S.A.

4 Osservatorio Astronomico di Brera, Via E. Bianchi 46, I-22055 Merate (CO), Italy

5 Dipartimento di Fisica, Sez. Astrofisica, Università di Milano, Via Celoria 16, I-20133 Milano, Italy

6 C.A.I.S.M.I C.N.R., Largo E. Fermi 5, I-50125 Firenze, Italy

7 Max-Planck-Inst. für Kernphysik, Bereich Astrophysik, Saupfercheckweg 1, Postfach 1039 80, D-69029 Heidelberg, Germany

8 Osservatorio Astronomico di Brera, Via Brera 28, I-20121 Milano, Italy

Received November 30, 1998; accepted March 8, 1999

\begin{abstract}
We present near-infrared, $H$-band $(1.65 \mu \mathrm{m})$, surface photometry of 174 spiral galaxies in the area of the Pisces-Perseus supercluster. The images, acquired with the ARNICA camera mounted on various telescopes, are used to derive radial profiles of surface brightness, ellipticities, and position angles, together with global parameters such as $H$-band magnitudes and diameters ${ }^{1}$. The mean relation between $H$-band isophotal diameter $D_{21.5}$ and the $B$-band $D_{25}$ implies a $B-H$ color of the outer disk bluer than 3.5 ; moreover, $D_{21.5} / D_{25}$ depends on (global) color and absolute luminosity. The correlations among the various photometric parameters suggest a ratio between isophotal radius $D_{21.5} / 2$ and disk scale length of $\sim 3.5$ and a mean disk central brightness $\simeq 17.5 H$-mag $\operatorname{arcsec}^{-2}$. We confirm the trend of the concentration in$\operatorname{dex} C_{31}$ with absolute luminosity and, to a lesser degree, with morphological type. We also assess the influence of
\end{abstract}

\footnotetext{
Send offprint requests to: gmorioarcetri.astro.it

* Based on observations at the TIRGO, NOT, and VATT telescopes. TIRGO (Gornergrat, $\mathrm{CH}$ ) is operated by CAISMICNR, Arcetri, Firenze. NOT (La Palma, Canary Islands) is operated by NOTSA, the Nordic Observatory Scientific Association. VATT (Mt. Graham, Az) is operated by VORG, the Vatican Observatory Research Group.

Correspondence to: gmorio@arcetri.astro.it

1 Table 3 and Fig. 4 are only available in electronic form at the CDS via anonymous ftp to cdsarc.ustrasbg.fr (130.79.128.5) or via http://cdsweb.ustrasbg.fr/Abstract.html. Radial profiles in tabular form and images FITS files are also available upon request from gmorio@arcetri.astro.it.
}

non-axisymmetric structures on the radial profiles and on the derived parameters.

Key words: galaxies: fundamental parameters - galaxies: spiral — infrared: galaxies

\section{Introduction}

In this paper we present near-infrared (NIR) $H$-band images of a sample of 174 spiral galaxies located in the region of the Pisces-Perseus (PP) supercluster, the largest lowredshift structure in the southern Galactic hemisphere. Although a number of measured infrared magnitudes exist in the literature for some clusters in this area (Aaronson et al. 1986), this is the first large NIR imaging survey of this structure and, together with the published surveys in the Coma supercluster (Gavazzi et al. 1996a,c) and in Virgo (Boselli et al. 1997), it makes up an extended database to investigate the properties of these most outstanding low-redshift superclusters and of their member galaxies. The data were collected to pursue several distinct lines of research: to probe the internal extinction in spiral disks (Moriondo et al. 1998c); to map peculiar motions in the supercluster (Baffa et al. 1993); to study stellar populations and dark matter content (Moriondo et al. 1998a,b).

As detailed in the following section, the galaxies observed pertain to two distinct sets: the first one was observed in the $H$-band only; the second one in three different passbands: $J, H$, and $K$, when possible. 


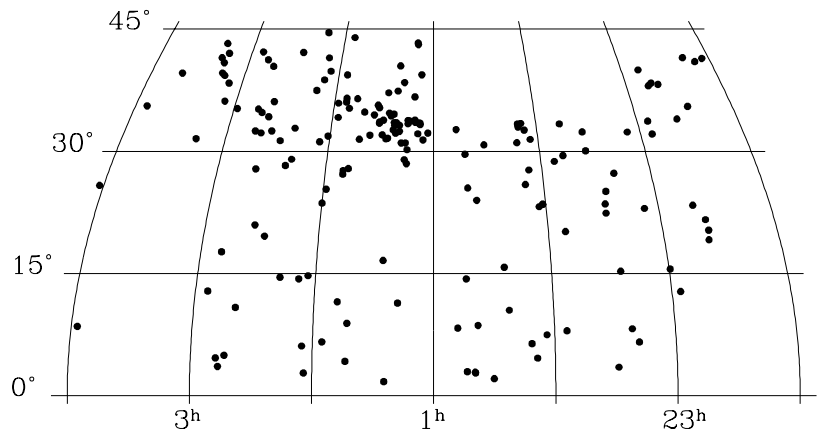

Fig. 1. Position of the sample objects in equatorial coordinates. In this equal-area projection, the clustering at $1^{\mathrm{h}} 20^{\mathrm{m}}$ and $33^{\circ}$ coincides with the Pisces cluster (sample B)

We defer the publication and analysis of the colour images to a forthcoming paper, and report here the $H$-band observations, that is the passband with the largest wealth of data, both in the literature and in our present data set. Although the resulting sample lacks completeness, the data are remarkably homogeneous both because of the type of objects included and for the uniformity in the observational and image reduction techniques. As it will be shown, the sample comprises a fair representation of the bright disk galaxies hosted in the entire supercluster.

\section{Sample}

\subsection{Sample selection}

The sample objects were selected from the catalogued disk galaxies in the Pisces-Perseus supercluster area, $22^{\mathrm{h}}<$ $\mathrm{RA}<4^{\mathrm{h}}$ and $0^{\circ}<\delta<45^{\circ}$, excluding obvious foreground members with $V_{\mathrm{H}}<3000 \mathrm{~km} \mathrm{~s}^{-1}$ and ellipticals. To best fit the camera fields of view to be employed, the sample was restricted to galaxies with optical major axis 0 . $5<a<4$.' 0 , and, among these, we further selected those with available good-quality $21-\mathrm{cm}$ spectra (Giovanelli \& Haynes 1989 and references therein). These selection criteria result into about 950 galaxies, mainly late spirals. From this set two distinct subsamples were extracted. Sample A comprises about 150 galaxies, randomly selected to cover the entire area and inclination range, and with types Sb or later; for these objects only $H$-band data were acquired. Sample B includes the 50 cluster galaxies with distance $d_{\mathrm{c}}<5.5$ from the Pisces cluster center $\left(1^{\mathrm{h}} 20^{\mathrm{m}}, 33^{\circ}\right)$, with optical size $D_{25} \geq 0$ ' 8 , and inclination $30^{\circ}<i<75^{\circ}$; for these objects, ranging in morphology from S0 to Sd, $J, H$, and $K$-band images were acquired.

Due to malfunctions and bad weather, not all the sample objects could be observed. As a whole 177 objects were observed at least in one band (usually $H$ ), 147 of which belong to the supercluster sample (sample A) and 35 to the cluster one (sample B); five galaxies are common to

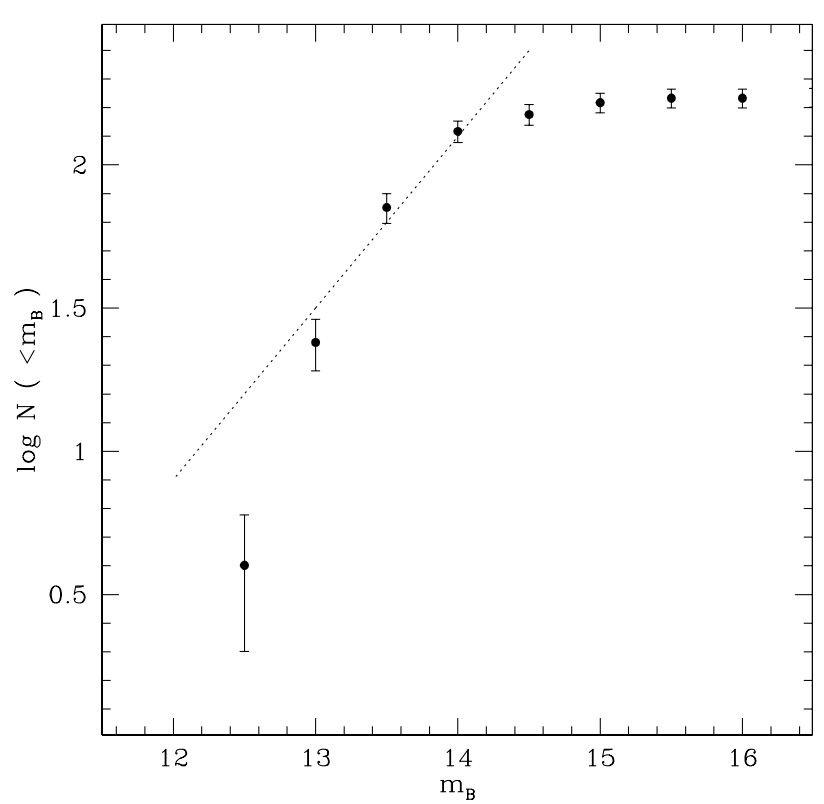

Fig. 2. Number of galaxies with apparent magnitude brighter than $m_{B}$ vs. $m_{B}$ ( $B_{\mathrm{T}}$ system). Error bars are $1 \sigma$ uncertainties due to counting statistics. The dotted line, shown for comparison, has the slope 0.6 expected for a uniform distribution in euclidean space; the position of the line is arbitrary

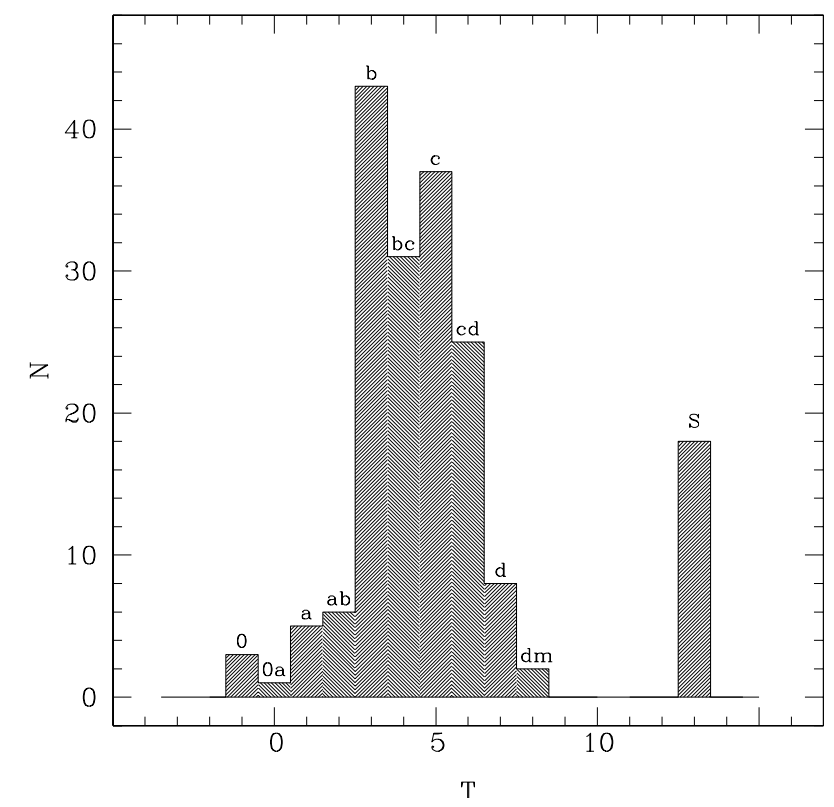

Fig. 3. Morphological distribution of the sample galaxies. $N$ is the number of objects per $\mathrm{RH}$ classification bin, $T$ is the index of stage along the Hubble sequence from the RC3. Spiral types are also reported on top of each bin. The rightmost bin, coded S, refers to objects classified S or SB without further specification 
Table 1. Extinction coefficients

\begin{tabular}{lrrr}
\hline Telescope & $J$ & $H$ & $K$ \\
\hline TIRGO & 0.083 & 0.029 & 0.063 \\
NOT & 0.057 & 0.000 & 0.042 \\
VATT & 0.067 & 0.007 & 0.040 \\
\hline
\end{tabular}

both samples. This paper presents data for the 174 galaxies imaged in the $H$ bandpass.

\subsection{Sample statistics}

The spatial distribution of the sample objects is depicted, in an equal-area projection, in Fig. 1. The region is bounded to the east by the Zone of Avoidance due to extinction in the Galactic plane. Despite its scarcity, our sample delineates the overall structure of the supercluster as portrayed by much richer sets (e.g. Giovanelli et al. 1986). The main ridge appears clearly, spanning the entire RA range between $30^{\circ}$ and $40^{\circ}$ declination, as well as the southern extensions down to the A400 and Pegasus clusters. Despite the presence of several rich clusters in the area, the only clear clustering is noted at the position of the Pisces cluster (NGC 507: $1^{\mathrm{h}} 20^{\mathrm{m}}, 33^{\circ}$ ); obviously this is due to the galaxies of sample $\mathrm{B}$.

In Fig. 2 we plot the cumulative distribution in photographic magnitude ( $B_{\mathrm{T}}$ system). When compared to the expectation for a uniformly distributed set, the sample starts deviating and losing completeness at $\sim 14 \mathrm{~B}$ mag; there is also a notable a lack of bright galaxies $\left(m_{B}<13.0\right)$. Both limits are mainly induced by the constraints on the apparent size and only to a minor degree by the characteristics of the UGC (Nilson 1973) and CGCG (Zwicky et al. 1961-68) catalogues, from which the sample was originally drawn; the scarcity of bright objects also follows the exclusion of foreground objects.

The morphological distribution is illustrated in Fig. 3. The types are drawn from the RC3 catalogue (de Vaucouleurs et al. 1991) where most of our sample galaxies have a detailed Revised Hubble ( $\mathrm{RH})$ classification; only 16 are classified S or SB without further specification, and only two non-UGC objects are not classified. As expected, due to the selection based on 21-cm observations, the sample mainly consists of late spiral types and peaks around $T=4$, that is Sbc.

\section{The data}

\subsection{Observations}

The sample galaxies were observed in the course of several observing campaigns, from December, 1992 until
February, 1997. Most of the data were collected at the TIRGO telescope (Zermatt, CH) in 1992, 1993, 1994 and 1997; some galaxies were observed at the NOT telscope (La Palma, Canary Islands) in 1995 and some at the VATT telescope (Mt. Graham, Az) in 1996. The instrument used at all telescopes was the Arcetri NIR camera ARNICA, equipped with a $256 \times 256$ pixel NICMOS3 detector (Lisi et al. 1993, 1996; Hunt et al. 1996). The plate scale was $0.97,0.55$, and 0.45 arcsec pixel ${ }^{-1}$ at the TIRGO, NOT and VATT respectively.

All the galaxies were observed by alternating frames on the source and on adjacent empty sky positions, integrating $\sim 1^{\mathrm{m}}$ in each position. Typical total integration times on source were $\sim 5^{\mathrm{m}}$ in all bands, yielding $(1 \sigma)$ surface magnitude limits of 20.8, 21.3, and 20.6 H-mag $\operatorname{arcsec}^{-2}$ at the TIRGO, NOT and VATT respectively (Hunt \& Mannucci 1998). With a typical sky brightness of 14 $H$-mag $\operatorname{arcsec}^{-2}$, these limits correspond to $0.1-0.2 \%$ of the "background".

\subsection{Data reduction}

All image reduction was performed with IRAF and the STSDAS packages ${ }^{2}$.

The flat fields for each source exposure were obtained by averaging the sky frames acquired immediately before and after it, upon removal from each sky frame of eventual stars in the field. The use of medians of larger numbers of sky frames as flat fields ("superflats"), despite their lower noise, turns out to be effective only in case of exceptional stability of the atmospheric emission. Due to the high sky brightness, the requirements on flatness for mapping the outer regions of galaxies are quite strict. On average the resulting flatness (low-spatialfrequency noise only) of our final images is about $0.01 \%$ $(1 \sigma)$, negligible relative to the high-spatial-frequency noise which amounts to $0.1-0.2 \%$ as noted in Sect. 3.1. Such image quality allows a substantial improvement in signal-to-noise of the elliptically-averaged profiles (see Sect. 3.4).

The - typically four - flat-fielded source frames were subsequently cleaned for bad pixels, registered, and rescaled by an additive term to a common median level. They were then combined to obtain the median frame, after clipping deviant values in each position on the detector. The final step was the subtraction of the background, which was estimated on the source frame itself upon automatic exclusion of star and galaxy pixels.

\footnotetext{
${ }^{2}$ IRAF is the Image Analysis and Reduction Facility made available to the astronomical community by the National Optical Astronomy Observatories, which are operated by AURA, Inc., under contract with the U.S. National Science Foundation. STSDAS is distributed by the Space Telescope Science Institute, which is operated by the Association of Universities for Research in Astronomy (AURA), Inc., under NASA contract NAS 5-26555.
} 
Table 2. Summary of observations

\begin{tabular}{|c|c|c|c|c|c|}
\hline $\begin{array}{l}\text { Telescope } \\
\text { (1) }\end{array}$ & $\begin{array}{l}\text { Date } \\
{[\mathrm{yy} / \mathrm{mm} / \mathrm{dd}]} \\
(2)\end{array}$ & $\begin{array}{l}\text { Zero Point } \\
{\left[\begin{array}{l}H \text { mag }] \\
(3)\end{array}\right.}\end{array}$ & $\begin{array}{c}\sigma_{\mathrm{ZP}} \\
{[H \mathrm{mag}]}\end{array}$ & $\begin{array}{l}F W H M \\
{[\operatorname{arcsec}]} \\
\quad(5)\end{array}$ & $\begin{array}{l}\text { Objects observed } \\
\qquad(6)\end{array}$ \\
\hline $\mathrm{T}$ & $92 / 12 / 27$ & 19.47 & 0.08 & 2.3 & UGC: 1205,1633 \\
\hline $\mathrm{T}$ & $93 / 10 / 16$ & 19.51 & 0.04 & 2.1 & UGC: $732,835,975$ \\
\hline $\mathrm{T}$ & $93 / 10 / 18$ & 19.26 & 0.20 & 2.6 & UGC: 646,820 \\
\hline $\mathrm{T}$ & $94 / 09 / 25$ & 19.74 & 0.05 & 3.5 & UGC: 673,919 \\
\hline $\mathrm{T}$ & $94 / 09 / 26$ & 19.76 & 0.08 & 2.7 & UGC: $690,11931,12527$ \\
\hline $\mathrm{T}$ & $94 / 09 / 27$ & 18.93 & 0.27 & 1.8 & UGC: $12098,12103,12234,12614$ \\
\hline $\mathrm{T}$ & $94 / 09 / 29$ & 19.76 & 0.11 & 2.1 & UGC: $98,100,438,484,725,12821,12915$ \\
\hline $\mathrm{T}$ & $94 / 11 / 16$ & 19.83 & 0.02 & 3.4 & UGC: 1688,1792 \\
\hline $\mathrm{T}$ & $94 / 11 / 18$ & 19.82 & 0.03 & 2.6 & $\begin{array}{l}\text { UGC: } 14,57,214,230,420,463,493,11973 \text {, } \\
12100,12137,12173,12586,12618\end{array}$ \\
\hline $\mathrm{T}$ & $94 / 11 / 21$ & 19.84 & 0.06 & 1.7 & $\begin{array}{l}\text { UGC: } 89,365,798,886,911,937,962,1094, \\
1259,1497,1629,2094,2241,12039,12108 \\
12230,12378,12486,12539,12610,12780\end{array}$ \\
\hline $\mathrm{T}$ & $94 / 11 / 24$ & 19.81 & 0.05 & 2.2 & $\begin{array}{l}\text { UGC: } 16,60,301,940,1238,1250,1292,2042 \text {, } \\
2109,2178,2200,12808,12834\end{array}$ \\
\hline $\mathrm{T}$ & $94 / 11 / 25$ & 19.85 & 0.04 & 2.1 & $\begin{array}{l}\text { UGC: } 728,796,857,906,909,1013,1302,1376, \\
\text { 1411, 1768, 2586, 2604, 2608, 2627, 2655, 2658, } \\
\text { 2885, 2931; CGCG 418-002 }\end{array}$ \\
\hline $\mathrm{T}$ & $94 / 11 / 26$ & 19.82 & 0.03 & 2.4 & $\begin{array}{l}\text { UGC: } 448,833,1005,1033,1034,1100,1111,1291 \text {, } \\
1582,1626,1835,2048,2142,2156,2353,2435, \\
2548,2810,11897,12153,12181,12199,12250, \\
12286,12379,12598,12666,12667 \text {; CGCG } 503-007\end{array}$ \\
\hline $\mathrm{T}$ & $94 / 11 / 27$ & 19.00 & 0.21 & 2.0 & $\begin{array}{l}\text { UGC: } 1210,1349,1695,1935,2134,2204,2223 \text {, } \\
2303,2368\end{array}$ \\
\hline $\mathrm{N}$ & $95 / 09 / 04$ & 21.12 & 0.03 & 1.1 & $\begin{array}{l}\text { UGC: } 26,74,114,311,414,462,714,927,1089, \\
1350,1355,1395,1451,1579,1805,2258,2264\end{array}$ \\
\hline $\mathrm{N}$ & $95 / 09 / 05$ & 21.10 & 0.04 & 1.1 & UGC: 697; CGCG: 501-091, 502-085, 502-097, 521-038 \\
\hline $\mathrm{N}$ & $95 / 09 / 08$ & 21.11 & 0.05 & 1.4 & UGC 745 \\
\hline $\mathrm{N}$ & $95 / 09 / 09$ & 21.06 & 0.04 & 0.9 & UGC 738, CGCG 502-021 \\
\hline $\mathrm{V}$ & $96 / 11 / 28$ & 20.41 & 0.05 & 1.2 & CGCG 502-032 \\
\hline $\mathrm{V}$ & $96 / 12 / 01$ & 20.43 & 0.01 & 1.8 & UGC: $77,776,12776$ \\
\hline $\mathrm{V}$ & $96 / 12 / 02$ & 20.45 & 0.02 & 1.4 & UGC: $106,380,1556,1564,1577$ \\
\hline $\mathrm{V}$ & $96 / 12 / 03$ & 20.46 & 0.02 & 1.9 & UGC: 800,831 \\
\hline $\mathrm{V}$ & $96 / 12 / 04$ & 20.35 & 0.04 & 0.9 & UGC 710 \\
\hline $\mathrm{T}$ & $97 / 02 / 03$ & 19.78 & 0.02 & 2.3 & UGC: $1887,1937,2617 ;$ CGCG 521-068 \\
\hline $\mathrm{T}$ & $97 / 02 / 04$ & 19.80 & 0.07 & 1.7 & UGC: 1131,2185 \\
\hline
\end{tabular}

\subsection{Photometric calibration}

A star from the list of northern standards by Hunt et al. (1998) was observed once about every hour in the three bands. In each band five frames were obtained with the star in different positions on the detector. A flat field for each standard star position was obtained using the "clipped" median of the remaining four positions.
Standard-star frames were also cleaned for bad pixels, but not averaged together. For every standard star observation, aperture photometry was performed in each position within a circle centered on the star, after subtracting the sky background. The radius of the aperture was typically 4 times the seeing $F W H M$; the background value was evaluated as the median in a circular annulus between radii 5 and $8 F W H M$. The instrumental 
magnitudes were (to first order) corrected for atmospheric extinction using the coefficients reported in Table 1 (from Hunt \& Mannucci 1998); units are mag airmass ${ }^{-1}$. An average zero point was then computed for each night of observations in every band and used to calibrate the relative galaxies. All the data presented here, magnitudes, surface magnitudes, and diameters, take into account only correction for atmospheric extinction; no correction has been applied for Galactic and internal extinction nor for the effects of redshift ( $\mathrm{K}$ correction, and angular size - $z$ relation). A summary log of the obervations is presented in Table 2, where for each observing night: Column 1 indicates the telescope used ( $\mathrm{N}$ for NOT, $\mathrm{T}$ for TIRGO, V for VATT); Column 2 is the date of the following day; Column 3 is the nightly averaged zero point in the $H$ band; Column 4 is the $1 \sigma$ uncertainty on the calibration as derived by the set of standard-star observations for the night; Column 5 is the average FWHM of the seeing measured on the standard-star images; Column 6 is the list of sample galaxies observed that night.

$H$-band multiaperture photometry of 11 of the sample galaxies is available from Bothun et al. (1985), who used a single-element, InSb photometer; the galaxies are UGC 646, 673, 732, 820, 919, 927, 1013, 1033, 1094, 12486, 12359. Similar data are also available from Baffa et al. (1993) for UGC 57, 60, 98, 975, 1094, 1100, 1302, 1411, $2548,12527,12666$. In order to compare these with our results, we have performed multiaperture photometry on our images using their aperture values, for a total of 24 measurements to compare with Bothun et al. and 55 with Baffa et al. The mean difference between the results by Bothun et al. and ours is $+0.04 \pm 0.08$ mag (standard deviation of the set, not of the mean), a quite comfortable result; the comparison with Baffa et al. yields a difference of $+0.07 \pm 0.14 \mathrm{mag}$. In neither case is a systematic difference clearly discernible ${ }^{3}$.

\subsection{Brightness profiles}

For each of the final galaxy images the coordinates of the center were determined by fitting a Gaussian to the center. Brightness profiles were then extracted by fitting elliptical contours of increasing galactic radius, keeping the center position fixed, with surface brightness $\mu_{\mathrm{H}}$, ellipticity $\epsilon=1-b / a$, and position angle PA as free parameters. The

\footnotetext{
${ }^{3}$ It may be noted that their observations were performed with a chopping technique and on-line sky subtraction. Due to the limited chopping throw, it is possible that external regions of the galaxy, as well as faint objects nearby, are included in the sky measurement with a consequent underestimate of the source flux within the aperture. Because of this, and of the difficulty of centering faint objects within the aperture diaphragms, single-photometer measurements are expected to be slightly fainter than those acquired by panoramic detectors.
}

profiles were sampled for increasing values of the major semiaxis $a$, from 1 to 10 pixels in steps of 1 pixel, and then outward with a $10 \%$ geometric progression $a_{i+1}=1.1 \times a_{i}$. Regions of the image containing close companions or foreground stars were edited out from the fitted area.

We also provide an estimate of the seeing $F W H M$ for each final image of a galaxy. This was determined in most cases using stars in the field of the galaxy image. When this was not possible, stars were selected in the images immediately before or after the one considered ${ }^{4}$. The resulting profiles for $\mu_{\mathrm{H}}, \epsilon$, and PA are reported to the right of the corresponding image in Fig. 4.

\section{Results}

The global photometric parameters are summarized in Table 3, whose entries are as follows.

Column 1: UGC number or, if missing, CGCG number;

Column 2: Other common names;

Column 3 and 4: Equatorial J2000 coordinates in standard units (hh mm ss.s, dd $\mathrm{mm}$ ss);

Column 5: Revised Hubble morphological type from the $\mathrm{RC} 3$;

Column 6: Total $B$ magnitude or, if missing, photographic magnitude reduced to the $B_{\mathrm{T}}$ system, from the RC3;

Column \%: Isophotal optical size, major $\left(D_{25}\right)$ and minor axes in arcmin at $25 \mathrm{~B}$-mag $\operatorname{arcsec}^{-2}$, from the RC3;

Column 8: Heliocentric systemic velocity in $\mathrm{km} \mathrm{s}^{-1}$ as listed in $\mathrm{NED}^{5}$, or, if missing, from private archives; Column 9: "Total" $H$ magnitude $\left(H_{\mathrm{T}}\right)$ within a circle of aperture $D_{25}$;

Column 10: Isophotal $H$ magnitude $\left(H_{21.5}\right)$ within the elliptical isophote at $21.5 \mathrm{H}$-mag $\operatorname{arcsec}^{-2}$;

Column 11: Major axis $\left(D_{21.5}\right)$ in arcmin of the elliptical isophote at $21.5 \mathrm{H}$-mag $\operatorname{arcsec}^{-2}$;

Column 12: Effective diameter $\left(D_{\mathrm{e}}\right)$ in arcmin; this is the major axis of the elliptical isophote containing half of the flux corresponding to $H_{\mathrm{T}}$;

Column 13: Concentration index $C_{31}$, defined as the ratio between the major axes of the ellipses enclosing $75 \%$ and $25 \%$ of the flux corresponding to $H_{21.5}$;

Column 14: Ellipticity $\epsilon=1-b / a$ of the outer elliptical isophotes;

Column 15: Position angle (PA) of the outer elliptical isophotes, computed Eastward from North;

Column 16: Telescope of observation: TIRGO (T), NOT $(\mathrm{N})$, and $\operatorname{VATT}(\mathrm{V})$;

\footnotetext{
${ }^{4}$ Obviously such estimates, given in Table 3, do not usually coincide with the nightly averaged $F W H M$ from standard stars listed in Table 2.

${ }^{5}$ This research has made use of the NASA/IPAC Extragalactic Database (NED) which is operated by the Jet Propulsion Laboratory, California Institute of Technology, under contract with the National Aeronautics and Space Administration.
} 
Column 1\%: Estimate ( $F W H M)$ of the seeing disk of observation in arcsec, see Sect. 3.4.

Column 18: Notes from the catalogues.

\subsection{Magnitudes}

Two magnitudes are here reported for the imaged galaxies: a total value $H_{\mathrm{T}}$ and an isophotal one $H_{21.5}$.

The total magnitude $H_{\mathrm{T}}$ measures the flux contained within a circular aperture the size of the optical diameter $D_{25}$. For our images this is always an extrapolated value and is computed with a procedure similar to the one outlined in Gavazzi \& Boselli (1996) and Gavazzi et al. (1996a), although the values here are not corrected for extinction and redshift. We estimate the average $1 \sigma$ accuracy of $H_{\mathrm{T}}$ to be $\sim 0.15 \mathrm{mag}$, half the error being contributed by noise and calibration and half by uncertainty in the extrapolation.

The isophotal magnitude $H_{21.5}$ is derived by integrating the surface brightness radial profile from the center out to the elliptical isophote at $21.5 \mathrm{H}$-mag $\operatorname{arcsec}^{-2}$. In some cases, due to insufficient field of view, or to a particularly noisy background, or to strong asymmetries, we were not able to fit elliptical contours down to such brightness levels. In these cases we provide an estimate of $H_{21.5}$ obtained by (exponential) extrapolation of the outer profile; extrapolated values are enclosed in parenthesis and constitute roughly $10 \%$ of the total. In practice, the extrapolation was performed by fitting a weighted linear regression to three outermost points of the surface-magnitude radial profile. In the few cases where such regression was deemed not satisfactory, the procedure was repeated with the 6 outermost points of the profile. We estimate the average $1 \sigma$ accuracy of $H_{21.5}$ to be $\sim 0.08 \mathrm{mag}$ in case of interpolation and twice as much for the extrapolated values. Again, such values are only corrected for atmospheric extinction.

As for the relation between the two types of magnitudes, we find $\left\langle H_{21.5}-H_{\mathrm{T}}\right\rangle=0.20$, exactly what was found for the sample in Gavazzi et al. (1996a). The relation between the two $H$ magntudes is illustrated in Fig. 5, where their difference is plotted vs. the magnitude itself and vs. the average $H$ surface magnitude. Given the already quoted accuracies for the two magnitudes, the distribution appears to consist of a normal range, for $H_{21.5}-H_{\mathrm{T}}<$ $0.3 \mathrm{mag}$, and by a deviant tail for the higher values. Such large differences are partly due to the inclusion of some faint spurious objects, such as foreground stars superimposed on the outer disk. In general, the points for the extrapolated values, that is when also $H_{21.5}$ had to be estimated by extrapolation of the brightness profile, are distributed similarly to the others, which implies that, as expected, most of the variance is contributed by $H_{\mathrm{T}}$. A significant correlation is evident between $\left(H_{21.5}-H_{\mathrm{T}}\right)$ and $H_{21.5}$ itself; upon inclusion of all points, the slope is $0.050 \pm 0.014$. A similar and tighter trend is detected in the dependence on $\left\langle\mu_{21.5}\right\rangle$, the average surface magnitude within the isophotal elliptical contour at $21.5 \mathrm{H}$-mag $\operatorname{arcsec}^{-2}$ (right panel). Such correlations are likely due to the narrow range of apparent diameters of the sample, see Sect. 2.1. This selection criterion causes the faintest galaxies to be often those with fainter surface brightness (and lower inclination) and, consequently, with smaller isophotal size and fainter isophotal magnitude.

We conclude that the accuracy in estimating our $H$ magnitudes, especially the total magnitudes $H_{\mathrm{T}}$, degrades for the faintest galaxies of the sample, and that an appreciable part of the error is systematic in the sense of yielding too bright $H_{\mathrm{T}}$ values for fainter $H_{21.5}$ and/or $\left\langle\mu_{21.5}\right\rangle$. Such a trend spells a word of caution for the use of heavily extrapolated magnitudes in, say, distance measurements such as the Tully-Fisher relation.

Since the difference $H_{21.5}-H_{\mathrm{T}}$ is ideally determined only by the outer disk, we note that, for an exponential disk with folding length $r_{\mathrm{d}}$, such difference depends only on $\rho=\left(D_{21.5} / 2\right) / r_{\mathrm{d}}$, the ratio between the isophotal radius and the folding one:

$H_{21.5}-H_{\mathrm{T}}=2.5 \log _{10}\left[\frac{1}{1-\mathrm{e}^{-\rho}(1+\rho)}\right]$,

if $H_{\mathrm{T}}$ is identified with the total, asymptotic magnitude of the disk. Also, if $\mu(0)$ is the disk central surface magnitude:

$\left[\left\langle\mu_{21.5}\right\rangle-\mu(0)\right]-\left(H_{21.5}-H_{\mathrm{T}}\right)=5 \log _{10}\left(\frac{\rho}{\sqrt{2}}\right)$.

In the left panel of Fig. 5, the dashed lines are curves of constant $\rho$, while in the right panel they are curves of constant $\mu(0)$. The average (outer) disk appears to have an isophotal radius $D_{21.5} / 2 \simeq 3.5 r_{\mathrm{d}}$, with a central brightness $\mu(0) \simeq 17.5 H$-mag $\operatorname{arcsec}^{-2}$.

\subsection{Diameters}

The $D_{21.5}$ diameter, reported in Col. 7 of Table 3 , is the major axis of the elliptical isophote at $21.5 \mathrm{H}$-mag $\operatorname{arcsec}^{-2}$. This is provided, in arcmin, for all the objects observed in the $H$ band. As for the isophotal magnitudes, in some cases $D_{21.5}$ had to be measured by (exponential) extrapolation of the outer profile; extrapolated values are enclosed in parenthesis. The average $1 \sigma$ accuracy of $D_{21.5}$ is usually about $5^{\prime \prime}$ and three times worse in case of extrapolation.

Figure 6 illustrates the comparison between our $D_{21.5}$ and the $B$-band $D_{25}$ from the RC3. In the left panel only $D_{21.5}$ values obtained by interpolation are reported and the data set has been divided into three bins of (optical) axial ratio $b / a$; the bin boundaries are those which result in bins with equal number of objects. Within the uncertainties, the relation $D_{21.5}$ vs. $D_{25}$ does not deviate from linearity. As a whole we find $D_{21.5}=0.89 D_{25}$, which implies that the $21.5 \mathrm{H}$-mag $\operatorname{arcsec}^{-2}$ is not as deep as the 


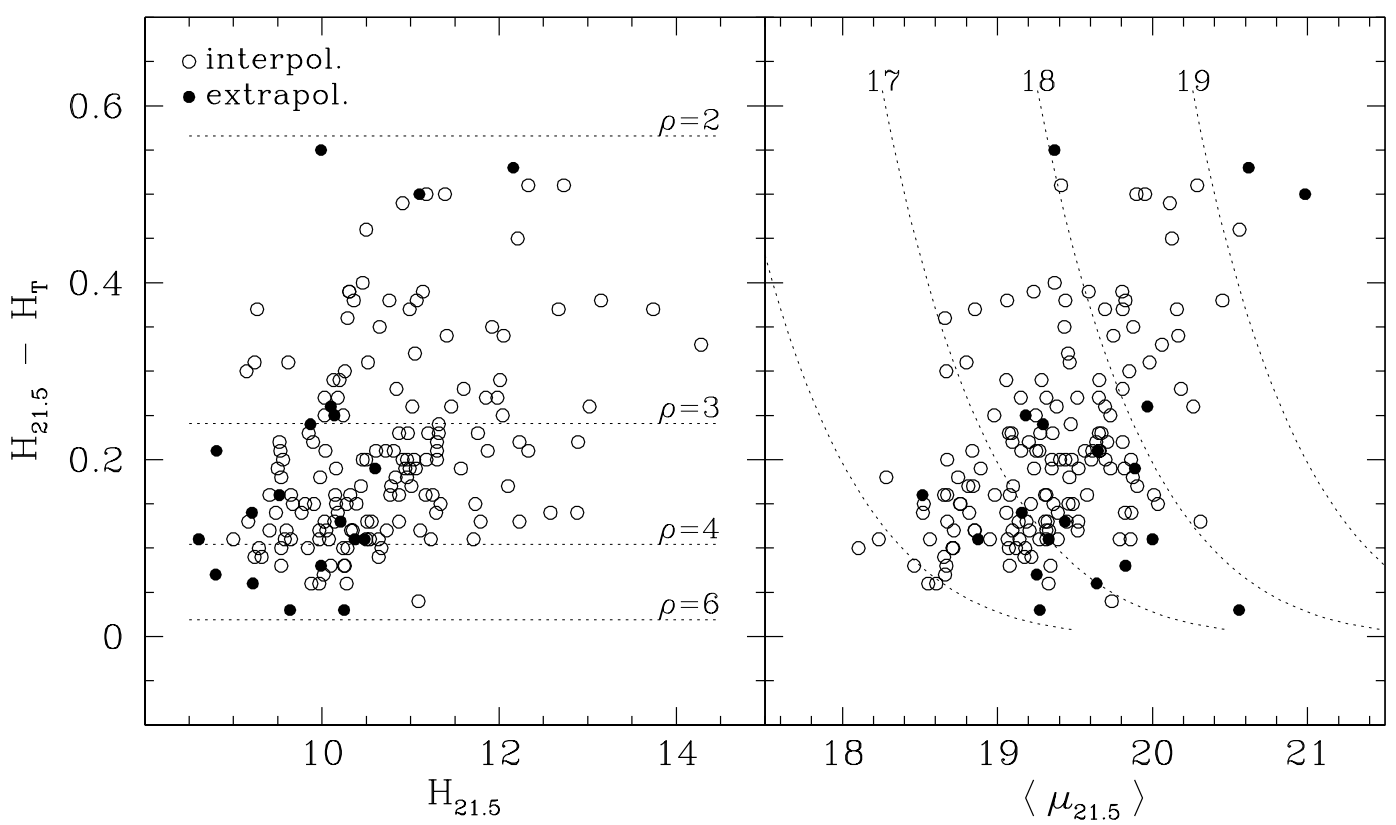

Fig. 5. The difference between the isophotal magnitude $H_{21.5}$ and the total magnitude $H_{\mathrm{T}}$ vs. $H_{21.5}$ (left panel), and vs. the average surface magnitude $\left\langle\mu_{21.5}\right\rangle$ (right panel). In both panels open symbols are used when the radial brightness profile extends to levels fainter than 21.5 (interpolation); solid symbols are used otherwise (extrapolation). In the left panel the dotted lines are the loci of constant $\rho$, the ratio of the isophotal radius $D_{21.5} / 2$ to the disk exponential folding length $r_{\mathrm{d}}$. In the right panel the dotted lines are loci of constant $\mu(0)$, the face-on disk surface brightness whose value in $H$-mag $\operatorname{arcsec}^{-2}$ is reported on top of each line

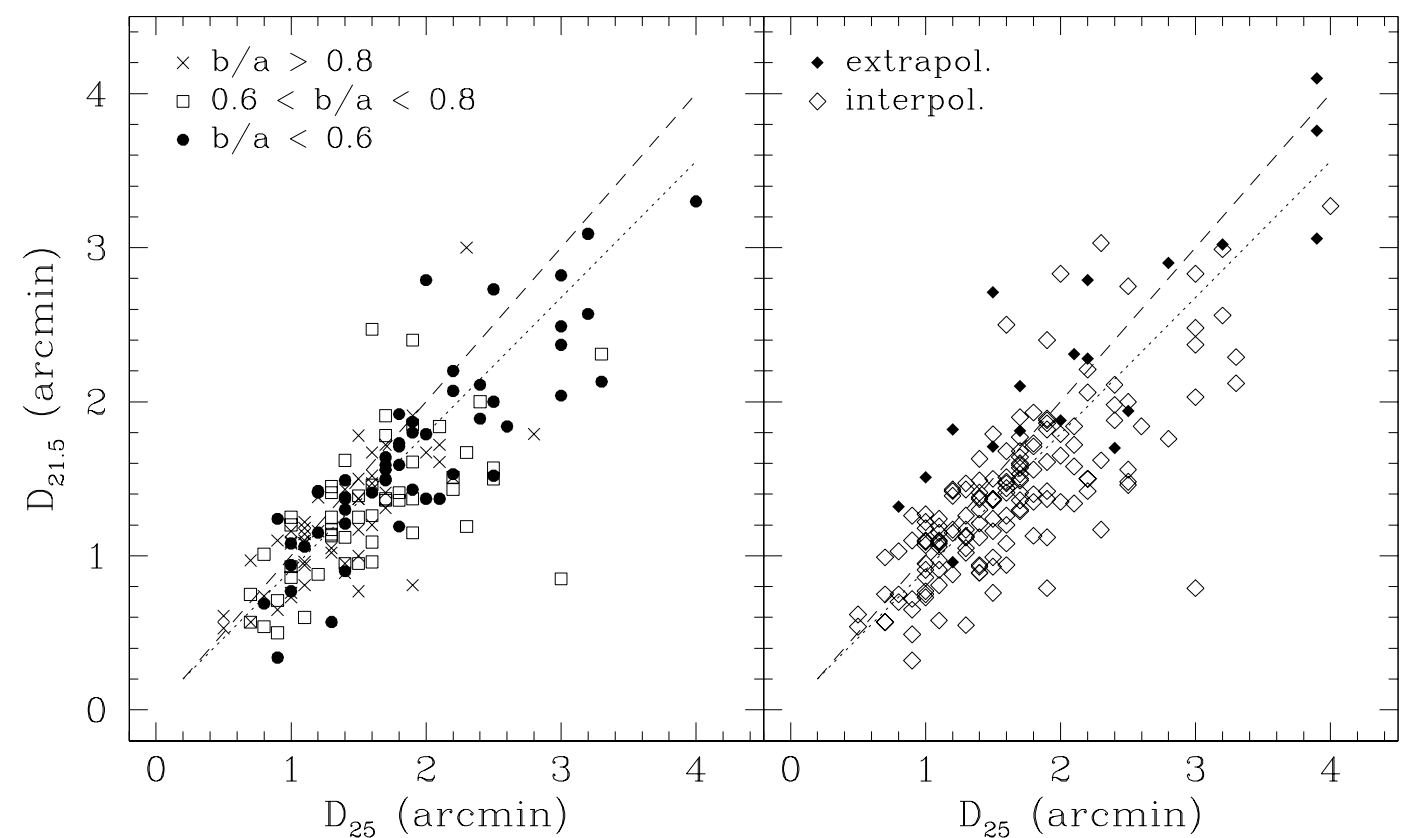

Fig. 6. The NIR isophotal diameter $D_{21.5}$ vs. the optical size $D_{25}$ from the RC3. In the left panel only $D_{21.5}$ values derived by interpolation are reported and the sample objects have been grouped into three classes according to the (optical) axial ratio $b / a$, and plotted with differerent symbols. In the right panel extrapolated and interpolated $D_{21.5}$ values are shown with different symbols. The dashed lines are for the case $D_{21.5}=D_{25}$ and the dotted ones for $D_{21.5}=0.89 D_{25}$, the average slope for the sample 


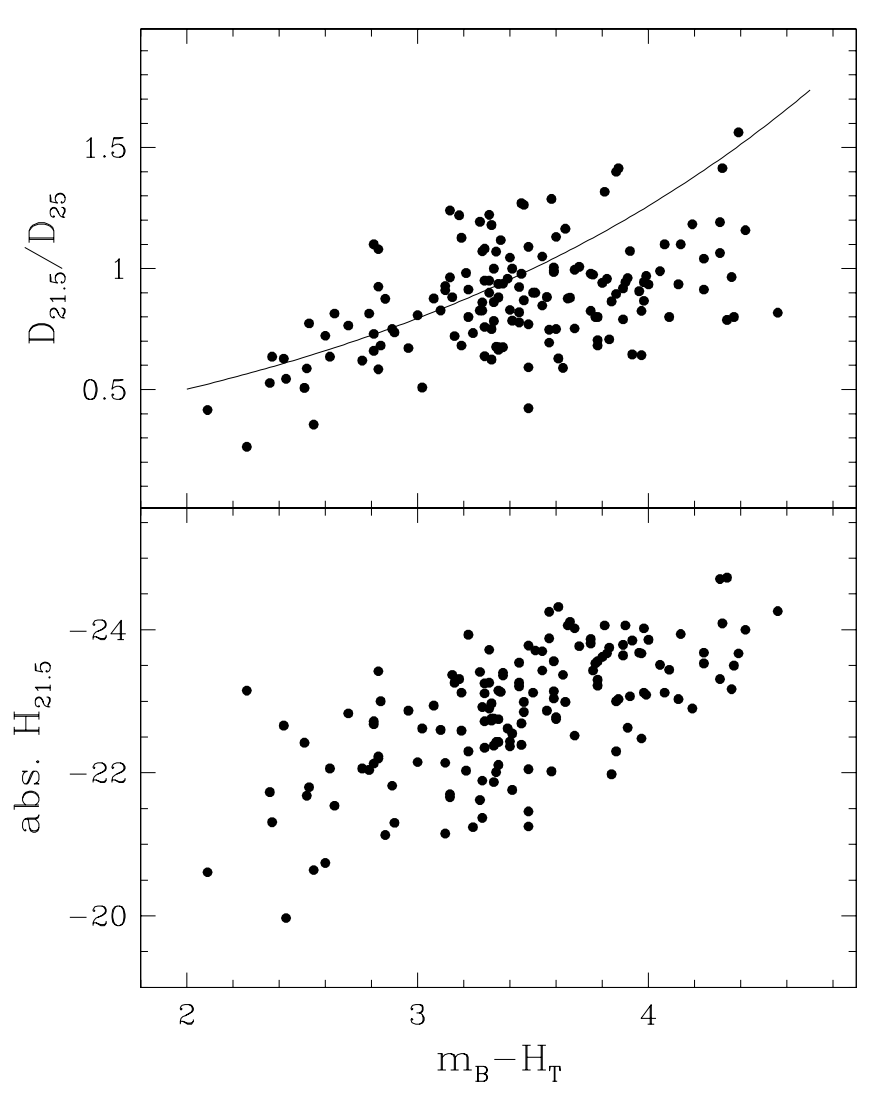

Fig. 7. The ratio between the isophotal $H$-band diameter $D_{21.5}$ and the $B$-band $D_{25}$ vs. the total $B-H$ colour $m_{\mathrm{B}}-H_{\mathrm{T}}$ (upper panel), and the colour-magnitude relation for the present sample (lower panel). Only the galaxies with $D_{21.5}$ measured by interpolation are reported. The line shown for comparison illustrates the behaviour of an exponential disk with fixed central surface magnitudes (see text)

standard $25.0 B$-mag $\operatorname{arcsec}^{-2}$; in other words, the $(B-H)$ colour of the outer galaxy regions is bluer, on average, than 3.5 (see also de Jong 1996). As for the comparison of the different inclination bins, we find no significant difference or trend: $D_{21.5}=0.88 D_{25}$ for $b / a<0.6, D_{21.5}=0.85 D_{25}$ for $0.6<b / a<0.8$, and $D_{21.5}=0.91 D_{25}$ for $b / a>0.8$. If any, the effects of internal extinction are not noticeable on this relation, which confirms the overall transparency of the outer disk. A last comment regards the rather large scatter about the average regression with $1 \sigma \simeq 30 \%$. This is true, and approximately constant, over the whole range of apparent size and does not depend on particularly deviant cases; as shown in the right panel of Fig. 6, it actually remains the same upon exclusion of the extrapolated $D_{21.5}$ values. The scatter can be attributed to the different methods of measurement: an objective estimate from the elliptically averaged profile in the case of $D_{21.5}$, and inspection of the 2D image for $D_{25}$. Especially in the case of late spirals, inspection of $B$-band plate material is strongly affected by spiral structure and by the presence of outer HII complexes. Further discussion of this issue will be found in Sect. 4.4.

The ratio between the isophotal optical and NIR diameters is found to be a weak function of the galaxy colour. This is illustrated in the upper panel of Fig. 7 where the ratio is plotted against the total $B-H$ index, $m_{B}-H_{\mathrm{T}}$. The solid curve represents an exponential disk with a central $B-H=3.5 \mathrm{mag}$ and different scale lengths in the two bandpasses; for this special value of the central colour $(3.5=25-21.5)$ the ratio $D_{21.5} / D_{25}$ is equal to the scale lengths ratio, and the total colour only depends on this ratio. Due to the well-known colour-magnitude relation (Tully et al. 1982), the dependence on colour also implies a certain dependence on the absolute luminosity; the colour-magnitude relation for our sample is shown in the lower panel of Fig. 7. The indicative absolute magnitudes are computed assuming a redshift distance with $H_{0}=$ $100 \mathrm{~km} \mathrm{~s}^{-1} \mathrm{Mpc}^{-1}$ and upon reduction of the velocity to the Local Group centroid according to RC3 (no infall correction); assuming a solar absolute magnitude of $3.39 H$-mag, as in Gavazzi et al. (1996b), the mean value of about $-22.5 \mathrm{mag}$ is equivalent to $2.310^{10} L_{\odot}$. No clear correlation was instead found between $D_{21.5} / D_{25}$ and the apparent parameters (magnitude, size, inclination) so that our $D_{21.5}$ estimates appears to be generally free of measurement biases.

The effective diameter $D_{\mathrm{e}}$ reported in Col. 12 of Table 3 is the major axis in arcmin of the fitted elliptical isophote containing half the flux corresponding to the total magnitude $H_{\mathrm{T}}$. The average $D_{\mathrm{e}}$ uncertainty, not including the error on $H_{\mathrm{T}}$ is about $2 \%$ but can be worse, up to $10 \%$, in case of peculiarly disturbed morphologies.

\subsection{Concentration indices}

The concentration index $C_{31}$, Col. 13 of Table 3 , is defined as the ratio between the major axes of the elliptical isophotes enclosing $75 \%$ and $25 \%$ of the flux corresponding to $H_{21.5}$ (Gavazzi et al. 1990). We have explored the possibility of biases in the measurement of $C_{31}$, by checking for correlations of the index with apparent magnitude, apparent size, and ellipticity, and found no evidence of any. As noted by Gavazzi et al. (1996a), $C_{31}$ depends on the galaxy absolute luminosity and, to a lesser degree, on morphological type; such relations, for the present sample, are illustrated in Fig. 8. As was the case also in Gavazzi et al. (1996a), the highest concentration indices are found in the most luminous Sb spirals. It also appears from Fig. 8 that the relation between $\log C_{31}$ and NIR absolute magnitude is not simply linear but definitely concave or $\mathrm{L}$ shaped. That is to say that, not only the mean value, but also the variance of the concentration index increases with luminosity. In Fig. 8 we indicate the value of $C_{31}$ of a pure 


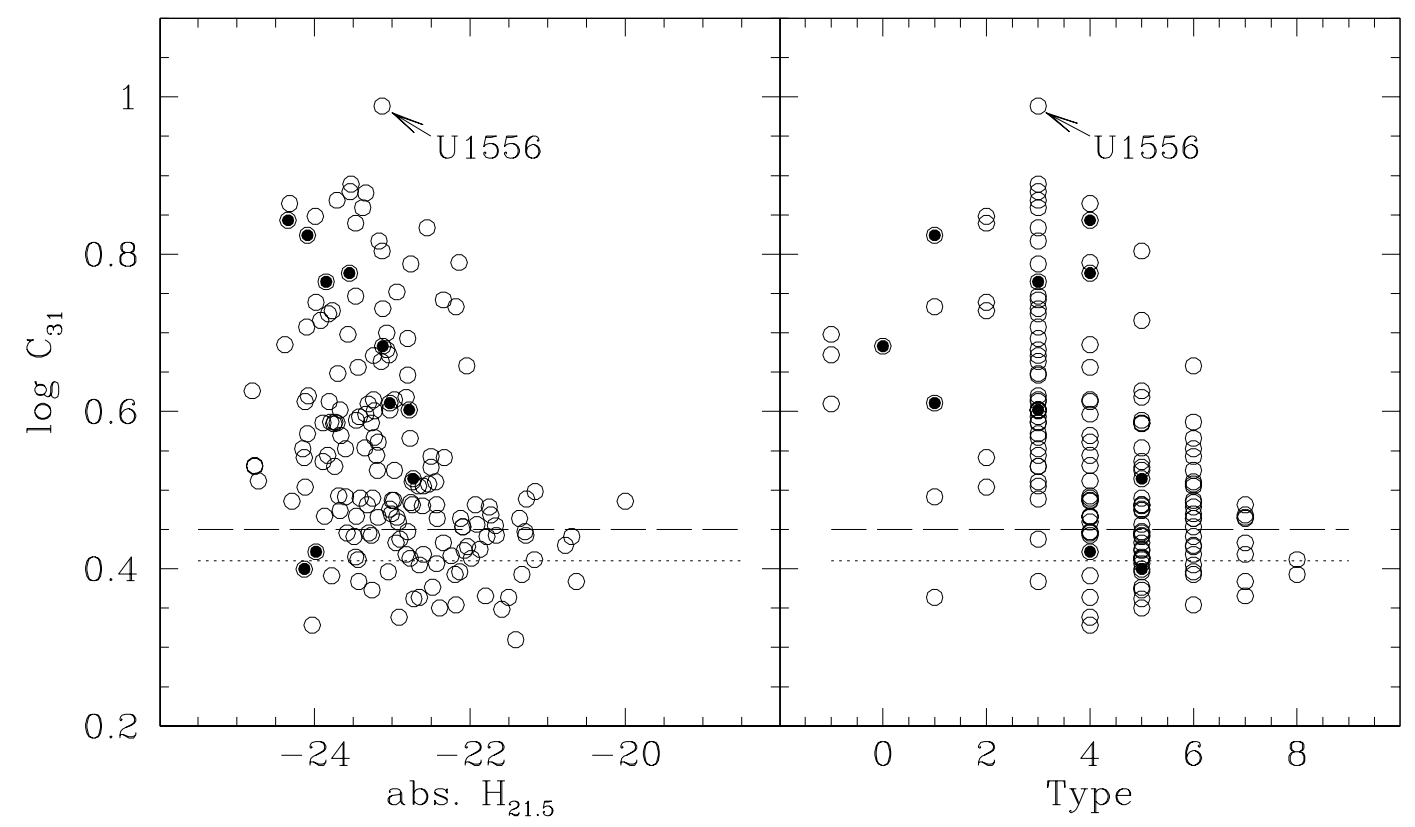

Fig. 8. $\log _{10}$ of the NIR light-concentration index $C_{31}$ vs. the absolute $H$-band magnitude (left panel), and vs. $T$, the index of stage along the Hubble sequence from the RC3 (right panel). Solid symbols are used for galaxies hosting active nuclei (Seyferts, LINERS, and starbursts). The horizontal lines identify the $C_{31}$ values of pure exponential disks (see text)

exponential disk: the upper value, $\log C_{31} \simeq 0.45$ is found when the $25 \%$ and $75 \%$ fractions refer to the total disk luminosity; the lower one, $\log C_{31} \simeq 0.41$, when the fractions refer to the luminosity within 3.5 exponential scale lengths, that is the average value corresponding to $H_{21.5}$ (see Sect. 4.1). Not surprisingly, there is a definite tendency of the lowest luminosities and latest morphologies to conform to a pure disk but some low concentration indices are present also among the luminous, early-type objects. A more detailed study of the characteristics of the bulge and disk components of these objects is deferred to forthcoming papers.

Ten of the sample galaxies, that is $\sim 6 \%$ of the total, are reported to host active nuclei, either Seyferts or LINERS or starbursts; they are marked with solid symbols in Fig. 8. While in the present sample they are quite luminous, there is no particular tendency to high $C_{31}$ values; also the distribution among the morphological types is rather uniform but for the avoidance at $T \geq 6$. We find no difference, for $C_{31}$, between the different classes of activity.

Figure 9 is a scatter diagram of the average surface brightness within the $21.5 \mathrm{H}$-mag $\operatorname{arcsec}^{-2}$ isophote versus $H_{21.5}$ and vs. $D_{21.5}$. While there is a definite correlation between $\left\langle\mu_{21.5}\right\rangle$ and $H_{21.5}$, in the sense that faint surface brightnesses are preferentially observed in the faintest sample objects, it disappears almost completely between $\left\langle\mu_{21.5}\right\rangle$ and $D_{21.5}$. The correlation is therefore determined by the limited range of the selected diameters (see Sect. 2.1) rather than by intrinsic properties. As for the isophotal diameters, the trend of $\left\langle\mu_{21.5}\right\rangle$ with inclination is not significant.

\subsection{Ellipticities and Position Angles}

The results of the elliptical-isophote fitting have been used to compute estimates of ellipticity $\epsilon$ and position angle PA of the outer regions. Our $\epsilon$ and PA values are computed directly from the output of the IRAF-STSDAS routine "ellipse" and are the weighted average of the three outermost points where the ellipticity is still evaluated with a precision better than 0.1 . The position angles are not reported for galaxies nearly face-on and particularly uncertain cases are flagged with a ":". We estimate the $1 \sigma$ uncertainty of the ellipticity to be $\sim 0.05$, in well-behaved cases.

It turns out that such a blind, although objective, procedure is often inaccurate. Indeed, an inspection of the radial profiles of $\epsilon$ and PA in Fig. 4 shows that they are often determined by the geometry of the spiral pattern, which often dominates even in the NIR, rather than by the effective orientation of the disk. This is true in particular for late spirals seen nearly face on and, obviously, for the more disturbed, peculiar morphologies. In addition our images have a restricted field of view and are somewhat shallower than the plates from which the optical values were estimated and therefore our estimate of the outer disk can be, in some cases, rather uncertain. As a consequence, the values we derive sometimes deviate considerably from those reported in the catalogues, which are also shown for comparison in Fig. 4. A direct comparison of our ellipticities, $\epsilon_{\mathrm{H}}$, and those from the RC3, $\epsilon_{\mathrm{B}}$, is shown in Fig. 10. As expected the scatter is rather large and not appreciably influenced by the most uncertain values; we count 25 galaxies out of 174 for which $\left|\epsilon_{\mathrm{H}}-\epsilon_{\mathrm{B}}\right|>0.2$. It is also 


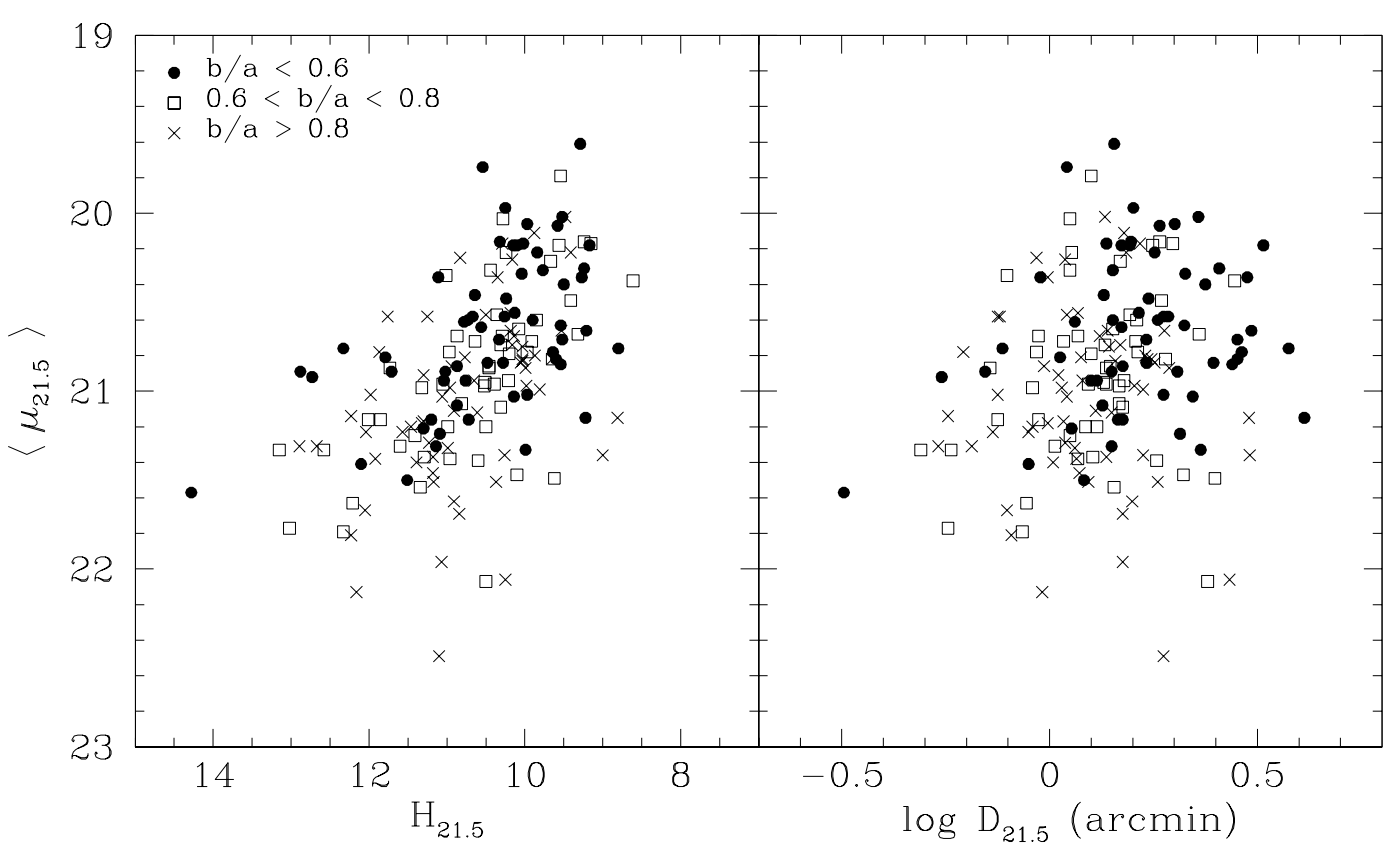

Fig. 9. $\left\langle\mu_{21.5}\right\rangle$, the average NIR surface brightness within the isophote at $21.5 H$-mag $\operatorname{arcsec}^{-2}$ vs. the apparent $H$ magnitude (left panel) and vs. the isophotal $D_{21.5}$ diameter (right panel). As in Fig. 6, different symbols refer to different ranges of optical axial ratios

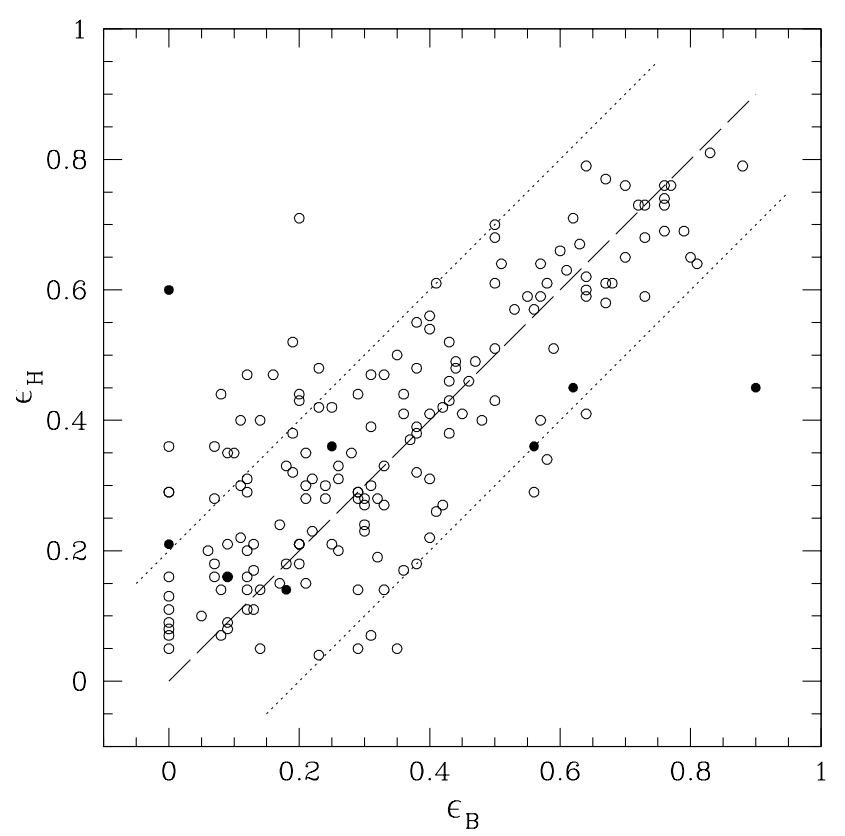

Fig. 10. The NIR ellipticity of the outer isophotes $\epsilon_{\mathrm{H}}$ vs. $\epsilon_{\mathrm{B}}$, the ellipticity listed in optical catalogues. Solid points refer to particularly uncertain $\epsilon_{\mathrm{H}}$ values. The dashed line is for the case $\epsilon_{\mathrm{H}}=\epsilon_{\mathrm{B}}$; the dotted lines enclose the region $\left|\epsilon_{\mathrm{H}}-\epsilon_{\mathrm{B}}\right|<0.2$

quite clear that most discrepant values are found for lowinclination objects, where $\epsilon_{\mathrm{H}}$ tends to be definitely larger than $\epsilon_{\mathrm{B}}$.

\subsection{Non-axisymmetric structures}

The fact that the elliptical-isophote fitting might occasionally be misled by non-axisymmetric structure is bound to bear an influence on the parameters derived by the brightness profile. For example, a strong bar on a very tenuous disk will induce artificially high values of the ellipticity; the result of such narrow isophotes will be larger isophotal diameters and consequently incorrect estimates of $H_{21.5}$ and $C_{31}$. To check the impact of such an effect we have selected the galaxies for which our estimates of $\epsilon$ and PA differ most from the catalogued values and have repeated the fitting procedure by imposing on the outer regions the $\epsilon$ values from the RC3. In most cases, as expected, such optical values favour rounder images with a consequent shrinking of $D_{21.5}$. The effect, in any case, is not dramatic and confined to less than $10 \%$, although it reaches over $30 \%$ in the two most deviant cases: UGC 1471 and 1626 . The effect on $H_{21.5}$ is in the sense of making it brighter at lower ellipticity, but rarely exceeds the errors associated with noise and calibration. Also the influence on $C_{31}$ is appreciable only in the worst cases and even here within $10 \%$; lower ellipticities tend to yield lower concentration indices.

In a certain number of cases, the isophotal fitting, although strongly influenced by non-axisymmetric structures in the inner regions, is able to recover the actual $\epsilon$ and PA of the outer disk. These cases $(\sim 20)$ are characterized by sudden and strong jumps in their $\epsilon$ and PA profiles. A good example is UGC 12039, which has both a strong bar and strong spiral arms. As shown in Fig. 11, the radial profiles of $\mu, \epsilon$, and $\mathrm{PA}$ all show an obvious jump 


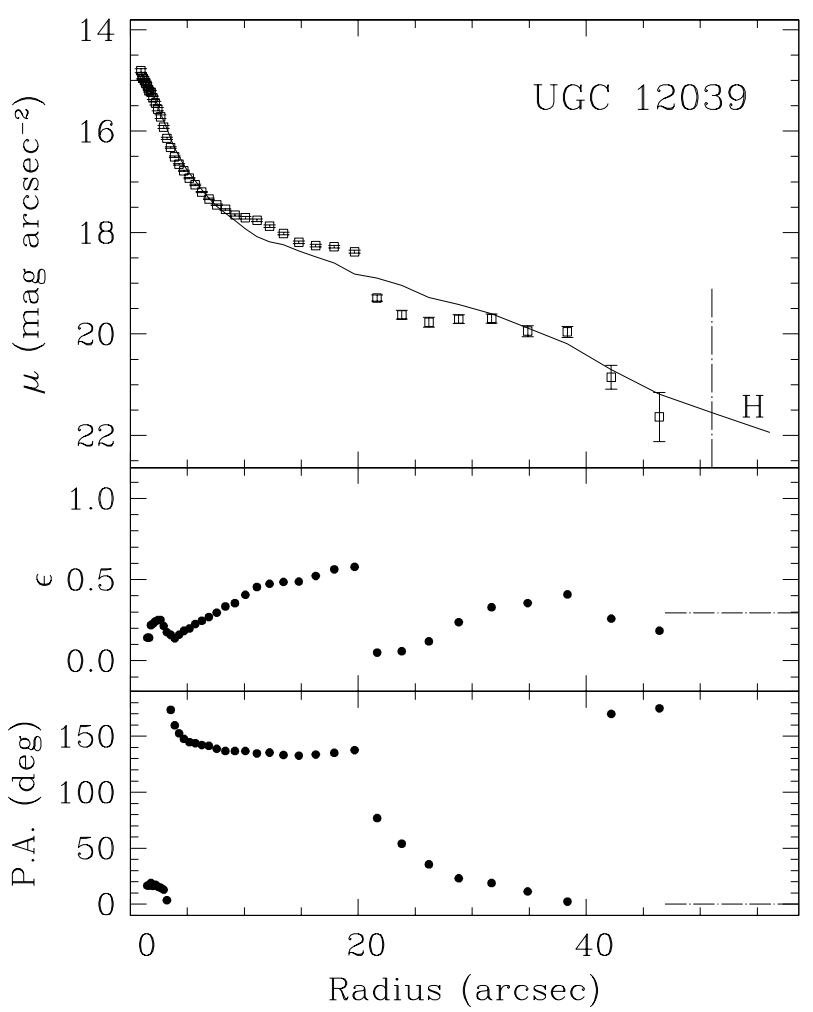

Fig. 11. Elliptically averaged radial profile of surface magnitude $\mu$, ellipticity $\epsilon$, and position angle PA for the barred $\mathrm{Sb}$ UGC 12039. The influence of the bar on the isophotal fitting between 5 and 20 arcsec is clearly illustrated in all three profiles. The solid line in the top panel represents the surface magnitude profile obtained by imposing at all radii a fixed $\epsilon=0.29$ and a fixed $\mathrm{PA}=0^{\circ}$, the average values of the outer disk

at $\sim 20$ arcsec, that is right after the fading of the bar. In the same figure we also show the $\mu$ profile obtained by imposing a fixed $\epsilon$ and PA value, 0.29 and $0^{\circ}$ respectively, which are the average values for the outer regions. The influence of the bar on the derived surface brightness profile is clearly depicted, with the strongest deviations reaching $\sim 0.5$ mag. However, in these cases, the global photometric parameters (magnitudes, diameters, indices) estimated in the two ways are virtually identical, with differences well within the limits of the quoted accuracy. For uniformity with the rest of the sample, and given the small bearing on the global parameters, we prefer to retain also in case of strong asymmetries the fitting procedure with free $\epsilon$ and PA. In turn this yields the observed $\epsilon$ and PA profiles, together with higher order azimuthal Fourier components of the luminosity distribution (Carter 1978), which will be used in a forthcoming paper of this series to investigate the properties of bars and non-axisymmetric structures in general.

\section{Summary}

We have presented the results of a programme of NIR imaging of disk galaxies in the Pisces-Perseus supercluster area, $22^{\mathrm{h}}<\mathrm{RA}<4^{\mathrm{h}}$ and $0^{\circ}<\delta<45^{\circ}$. This programme constitutes the first large NIR imaging survey of the supercluster and complements previous similar surveys in the Coma and Virgo areas. The present paper is the first of a series and contains images in the $H$ passband of a composite sample of 174 galaxies.

1. The sample galaxies, a subset of the PP supercluster galaxies, are mainly late spirals with a median $T \sim 5$. The sample starts lacking completeness at $B_{\mathrm{T}}=14.0$, and extends up to $B_{\mathrm{T}}=16.5$.

2. For each galaxy we present a grey-scale image reaching an isophote of $\sim 21.0 \mathrm{H}$-mag $\operatorname{arcsec}^{-2}$. For each image we supply the elliptically-averaged radial profile of the surface brightness, the ellipticity, and the position angle of the isophotes.

3. We derive a total magnitude $H_{\mathrm{T}}$, an isophotal magnitude $H_{21.5}$, an effective diameter $D_{\mathrm{e}}$, an isophotal one $D_{21.5}$, the concentration index $C_{31}$, and estimate the ellipticity $\epsilon$ and the position angle PA of the outer disk. These data, together with basic information regarding the sample objects and their observation, are collected in a single table which is available in electronic form at the CDS. Radial profiles in tabular form and images FITS files are also available upon request.

4. The trends of $\left(H_{21.5}-H_{\mathrm{T}}\right)$ with $H_{21.5}$ and $\left\langle\mu_{21.5}\right\rangle$ imply that the average outer disk has an isophotal radius $D_{21.5} / 2 \simeq 3.5 r_{\mathrm{d}}$ and a central surface brightness $\mu(0) \simeq 17.5 \mathrm{H}$-mag $\operatorname{arcsec}^{-2}$.

5. The empirical relation in our sample, $D_{21.5}=0.89 D_{25}$, implies that the $B-H$ color of the outer disk is bluer, on average, than 3.5, in agreement with de Jong (1996). The ratio $D_{21.5} / D_{25}$ depends, although weakly, on the total galaxy $B-H$, and consequently on its absolute magnitude.

6. We confirm the dependence of the (model independent) concentration index $C_{31}$ on luminosity and, to a lesser degree, on morphological type. Large values for $C_{31}$, that is values in excess of what is expected for pure disks $\left(\log C_{31} \gtrsim 0.5\right)$, are preferentially observed in galaxies brighter than $M_{\mathrm{H}} \simeq-23$ and $T \lesssim 3$.

7. Even in the NIR, objective estimates of outer ellipticities and position angles can often be contaminated by the geometry of bars and spiral patterns; the effect is strongest for shallow images, and for low inclinations. Their impact on the measurement of global photometric properties is, in any case, not dramatic. It virtually affects only $D_{21.5}$, with discrepancies exceeding $10 \%$ only in the two worst cases.

Acknowledgements. G.M. warmly acknowledges the hospitality of the Astronomy Dept. of Cornell University, where part 
of the data reduction was done. We acknowledge the partial support of the Italian Space Agency (ASI) through the grant ARS-98-116/22.

\section{References}

Aaronson M., Bothun G., Mould J., Huchra J., Schommer R.A., Cornell M.E., 1986, ApJ 302, 536

Baffa C., Chincarini G., Henry R.B.C., Manousoyanaki J., 1993, A\&A 280, 20

Boselli A., Tuffs R.J., Gavazzi G., Hippelein H., Pierini D., 1997, A\&AS 121, 507

Bothun G.D., Aaronson M., Schommer B., et al., 1985 ApJS 57,423

Carter D., 1978, MNRAS 182, 797

de Jong R.S., 1996, A\&A 313, 377

Gavazzi G., Boselli A., 1996, Ap. Lett. Comm. 35, 1

Gavazzi G., Garilli B., Boselli A., 1990, A\&AS 83, 399

Gavazzi G., Pierini D., Baffa C., et al., 1996a, A\&AS 120, 521

Gavazzi G., Pierini D., Boselli A., 1996b, A\&A 312, 397

Gavazzi G., Pierini D., Boselli A., Tuffs R.J., 1996c, A\&AS 120,489
Giovanelli R., Haynes M.P., 1989, AJ 97, 633

Giovanelli R., Haynes M.P., Chincarini, G., 1986, ApJ 300, 77 Hunt L.K., Mannucci F., 1998, Arcetri Technical Report $1998 / 2$

Hunt L.K., Lisi F., Testi L., et al., 1996, A\&AS 115, 181

Hunt L.K., Mannucci F., Testi L., et al., 1998, AJ 115, 2594

Lisi F., Baffa C., Hunt L.K., 1993, in Proceedings of SPIE Conference 1946, p. 594

Lisi F., Baffa C., Biliotti V., et al., 1996, PASP 108, 364

Moriondo G., Giovanardi C., Hunt L.K., 1998a, A\&AS 130, 81

Moriondo G., Giovanardi C., Hunt L.K., 1998b, A\&A 339, 409

Moriondo G., Giovanelli R., Haynes M.P., 1998c, A\&A 338, 795

Nilson P., 1973, Uppsala General Catalogue of Galaxies, Roy. Soc. Sci. Uppsala, Uppsala (UGC)

Tully B., Mould J., Aaronson M., 1982, ApJ 257, 527

de Vaucouleurs G., de Vaucouleurs A., Corwin H.G., et al., 1991, Third Reference Catalogue of Bright Galaxies (3 volumes). Springer-Verlag, New York (RC3)

Zwicky F., Herzog E., Kowal C.T., Wild P., Karpowicz M., 1961, 1963, 1965, 1966, 1968a,b, Catalogue of Galaxies and of Clusters of Galaxies (6 volumes). Calif. Inst. Techn., Pasadena (CGCG) 